\title{
Large Area, Low Capacitance Si(Li) Detectors for High Rate X-Ray Applications
}

C.S. Rossington, P.M. Fine and N.W. Madden

Engineering Science Department

Engineering Division

Lawrence Berkeley Laboratory

University of California, Berkeley CA, 94720

October 1992

This work was supported by the Director, Office of Energy Research, Office of Biological and Environmental Research, Analytical Technology Division of the U.S. Department of Energy under Contract Number DE-AC03-76SF00C98.

\section{innurieil}

DISTRIGUTION OF THIS ONCUMENT IS UNLIMITE. 


\title{
Large Area, Low Capacitance $\mathrm{Si}(\mathrm{Li})$ Detectors for High Rate X-Ray Applications
}

\author{
C. S. Rossington, P. M. Fine and N. W. Madden \\ Engineering Science Department, Engineering Division ${ }^{1}$ \\ Lawrence Berkeley Laboratory, University of California, Berkeley, CA, 94720 USA
}

\section{Abstract}

Large area, single-element $\mathrm{Si}(\mathrm{Li})$ detectors have been fabricated using a novel geometry which yields detectors with reduced capacitance and hence reduced noise at short amplifier pulse-processing times. A typical device employing the new geometry with a thickness of $6 \mathrm{~mm}$ and an active area of 175 $\mathrm{mm}^{2}$ has a capacitance of only $0.5 \mathrm{pf}$, compared to $2.9 \mathrm{pf}$ for a conventional planar device with equivalent dimensions. These new low capacitance detectors, used in conjunction with low capacitance field effect transistors, will result in $x$-ray spectrometers capable of operating at very high count rates while still maintaining excellent energy resolution. The spectral response of the low capacitance detectors to a wide range of $x$-ray energies at $80 \mathrm{~K}$ is comparable to typical stateof-the-art conventional $\mathrm{Si}(\mathrm{Li})$ devices. In addition to their low capacitance, the new devices offer other advantages over conventional detectors. Detector fabrication procedures, I-V and $\mathrm{C}-\mathrm{V}$ characteristics, noise performance, and spectral response to $2-60 \mathrm{keV} x$-rays are described.

\section{INTRODUCTION}

Low noise, high energy resolution $x$-ray detectors are commonly fabricated from lithium-drifted silicon or high purity germanium with dimensions of the order of $5-10 \mathrm{~mm}$ in diameter and 5-10 $\mathrm{mm}$ in thickness. These small, low capacitance detectors are then matched with low capacitance field effect transistors (FETs) in the $x$-ray spectrometers. The energy resolution of such spectrometers, at amplifier pulseprocessing times of less than approximately $20 \mu \mathrm{s}$, can be modeled after a simple CR-RC filter, as shown below [1]:

$$
\Delta E=\frac{\left(C_{d}+C_{f}+C_{s}\right) \varepsilon B 2.35}{q \sqrt{T_{p}}}
$$

where $\triangle E$ is the electronic component of the energy resolution in $\mathrm{eV}, \mathrm{C}_{\mathrm{d}}$ is the detector capacitance in farads, $\mathrm{C}_{\mathrm{f}}$ is the FET capacitance, $C_{s}$ is any stray capacitance arising from the capacitive coupling of spectrometer components in close proximity to the detector and FET, $\varepsilon$ is the ionization constant for the detector material in $\mathrm{eV}$ per electron-hole-pair, $\mathrm{B}$ is the channel noise of the FET measured in $V / \sqrt{\mathrm{Hz}}, q$ is the

\footnotetext{
${ }^{1}$ This work was supported by the Director, Office of Energy Research, Office of Biological and Environmental Research, Analytical Technology Division, of the U.S. Department of Energy under Contract No. DE-AC03-76SF00098.
}

electronic charge in Coulombs, and $T_{p}$ is the amplifier peaking time in seconds. For example, using typical values of $\mathrm{T}_{\mathrm{p}}=10 \mu \mathrm{s}, \mathrm{C}_{\mathrm{d}}=1 \mathrm{pf}, \mathrm{C}_{\mathrm{f}}=3.5 \mathrm{pf}, \mathrm{C}_{\mathrm{s}}=0.5 \mathrm{pf}, \mathrm{B}=1.5 \mathrm{x}$ $10^{-9} \mathrm{~V} / \sqrt{\mathrm{Hz}}$, and $\varepsilon=3.76 \mathrm{eV}$ for $\mathrm{Si}$ at $77 \mathrm{~K}$, then $\Delta \mathrm{E}$ is equal to $131 \mathrm{eV}$. (The total energy resolution, including both the electronic component and the detector component, would be equal to approximately $160 \mathrm{eV}$ for photons of $5.9 \mathrm{keV}$ ).

It is obvious from the equation above that the energy resolution could be improved by decreasing the capacitances of the detector and FET, and by increasing the peaking time of the amplifier. However, all of these options present limitations: To date, the lowest capacitance FETs available commercially for $\mathrm{x}$-ray spectrometers are $-2 \mathrm{pf}^{2}$, while values of 3-4 pf are more typical. Reducing the capacitance of the detector would require decreasing the detector area (which may already be smaller than desired for many practical applications) or increasing the detector thickness (which is typically already at its maximum). The peaking time of the amplifier can be increased to only approximately $20 \mu \mathrm{s}$ before the noise in the system is dominated by a noise component other than that modeled in equation (1). In addition, many experiments require that the pulse-processing time of the amplifier be as short as possible in crder to accommodate high count rate conditions.

Despite the restrictions imposed on the energy resolution as just described, there is a need for $\mathbf{x}$-ray spectrometers that can operate at high count rates and short amplifier pulseprocessing times while still maintaining excellent energy resolution, particularly for applications involving high intensity synchrotron radiation sources. Conversely, there are applications which do not require short pulse processing times, but do require detectors with large areas for collecting weak photon signals, while still maintaining excellent energy resolution. However, increasing the area of the detector has traditionally resulted in an increase in detector capacitance, which in turn degrades the energy resolution. In this paper, we describe the development and characterization of a large area, low capacitance $\mathrm{Si}(\mathrm{Li})$ detector ${ }^{3}$ which addresses these general requirements for high count rate operation and excellent energy resolution, and which offers significant advantages over the conventional $\mathrm{Si}(\mathrm{Li})$ detectors currently available for high energy resolution $\mathrm{x}$-ray spectroscopy.

\section{DETECTOR FABRICATION}

The large area, low capacitance (LALC) $\mathrm{Si}(\mathrm{Li})$ detectors were fabricated into the structure shown schematically in Fig.

\footnotetext{
2Interfet NJ14 FET.

${ }^{3}$ Patent pending.
} 
(a)

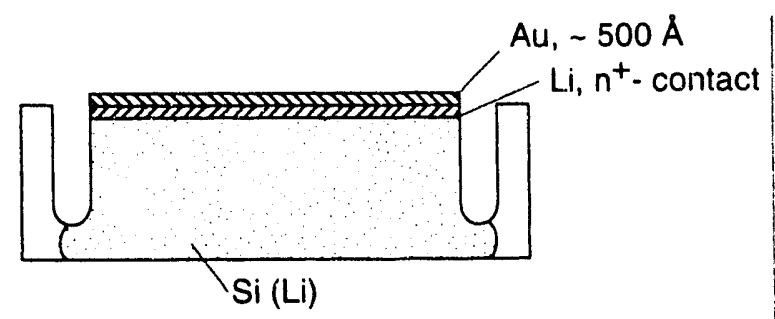

(b)
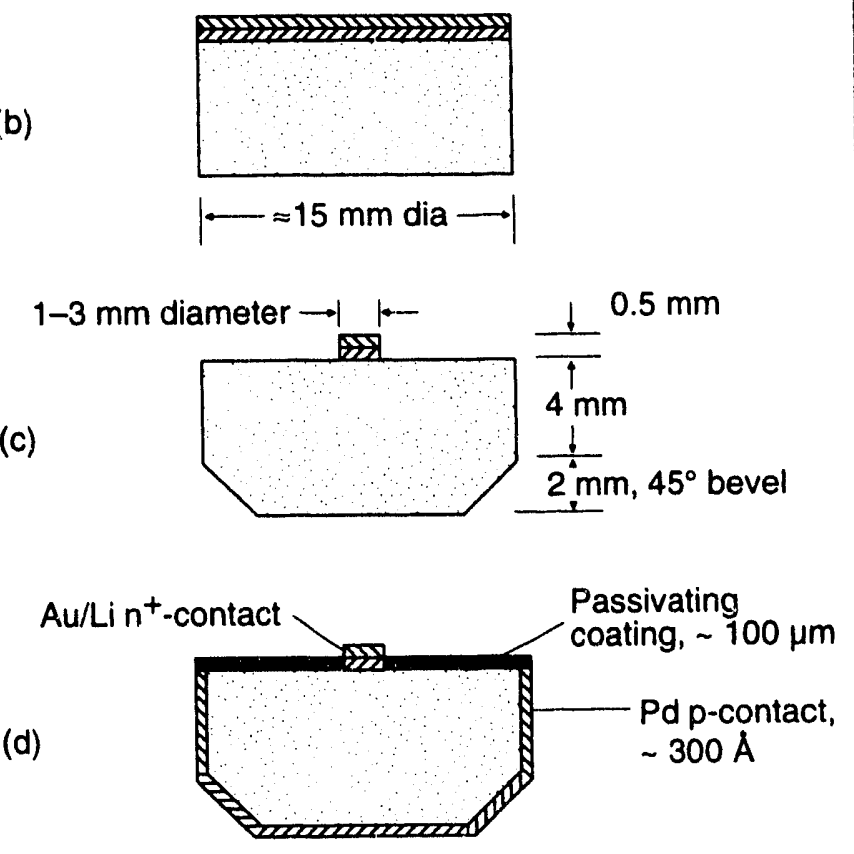

XBL. $923-5240$

Fig. 1 Processing sequence for the fabrication of the LALC detectors; details are described in the text.

(a) Conventional lithium-drifted silicon grooved structure.

(b) Cylinder for the low capacitance device cored out of center.

(c) Formation of the anode and bevel.

(d) Application of the Pd p-contact and the passivating coating.

1d. The geometry employed here is similar to that used in the past for high purity germanium detectors $[3,4]$. However, adapting this geometry to $\mathrm{Si}(\mathrm{Li})$ detectors has previously been difficult due to the restrictions imposed by the lithium-drifting process, the problems associated with passivating bare $\mathrm{Si}(\mathrm{Li})$ surfaces, and the inability to utilize any high temperature process once the Si material had been lithium-drifted. These limitations were overcome as described in the following fabrication sequence and illustrated in Fig. 1: High resistivity p-type silicon material (1000-2000 $\Omega$-cm) was compensated using the standard lithium-drifting technique in the conventional "grooved" geometry [2] (Fig. 1a). A cylindrical piece of the desired diameier for the LALC detector was then cored out of the center using an ultrasonic core-driller with $\mathrm{SiC}$ slurry (Fig. 1b). Approximately $0.5 \mathrm{~mm}$ of the original lithium-diffused surface was removed in the process of creating the protruding anode structure, which ranged from $1-3 \mathrm{~mm}$ in diameter (Fig. 1c). On some of the detectors, a $45^{\circ}$ bevel, approximately $2 \mathrm{~mm}$ deep, was cut from the bottom surface. On other detectors, the corners were radiused by removing $\sim 0.1$ $\mathrm{mm}$ of material by hand. After appropriate cleaning and etching of the bare $\mathrm{Si}(\mathrm{Li})$ surfaces, Pd was deposited for the surface barrier p-contact (which acts as the entrance window for the signal photons). A polyimide coating ${ }^{4}$ was then applied to passivate the bare $\mathrm{Si}(\mathrm{Li})$ surfaces of the LALC detectors (Fig. 1d). The coating was painted onto the freshly etched $\mathrm{Si}(\mathrm{Li})$ surface and then baked in air at $120^{\circ} \mathrm{C}$ for 25 minutes. This low temperature process did not affect the uniformity of the lithium compensation of the $\mathrm{Si}(\mathrm{Li})$ material, and the detectors did not require any additional lithium-drifting following this heat treatment.

The effects of the passivating coating on detector characteristics were tested on conventional $\mathrm{Si}(\mathrm{Li})$ detectors prior to using the coating on the LALC devices. Detector leakage current, capacitance and electronic noise were measured at $80 \mathrm{~K}$ on conventional $\mathrm{Si}(\mathrm{Li})$ detectors (similar to that shown in Fig. 1a), with and without the polyimide coating. Unlike other materials we have used in the past to passivate $\mathrm{Si}(\mathrm{Li})$ detectors, this particular coating did not measurably add to the capacitance, noise or leakage current of the conventional devices at $80 \mathrm{~K}$. In addition, other coatings we have used in the past to passivate surfaces of detectors with small anode structures produced surfaces which were not entirely electronically neutral and resulted in a sev e degradation of the peak/background ratio of the device. Although we have not yet measured the exact nature of the Si surface states created by this new passivating coating, it appears the surfaces are very neutral, as indicated by the excellent spectral performance of the detectors, which is detailed later.

Detectors were made with diameters in the range of 10-24 $\mathrm{mm}$, thicknesses of 4-6.3 mm, and anode diameters of 1-3 $\mathrm{mm}$. Table 1 lists the dimensions of the various devices that were fabricated and tested.

Table 1. Detector Dimensions

$\begin{array}{lccccc}\text { Detector } & \begin{array}{c}\text { O. D. } \\ (\mathrm{mm})\end{array} & \begin{array}{c}\text { Thickness } \\ (\mathrm{mm})\end{array} & \begin{array}{c}\text { Anode } \\ \text { Diameter } \\ (\mathrm{mm})\end{array} & \text { Bevel } & \begin{array}{c}\text { Detector } \\ \text { Capacitance } \\ (\mathrm{pf})\end{array} \\ 6911 & 10.8 & 4.2 & 2.5 & \text { yes } & 0.75 \\ 6913 & 11.6 & 4.3 & 2.9 & \text { no } & 0.80 \\ 7103 & 11.8 & 4.0 & 1.9 & \text { no } & 0.55 \\ 7505 & 11.0 & 3.9 & 2.6 & \text { no } & 0.75 \\ 7703 & 23.4 & 6.3 & 2.4 & \text { yes } & 0.65 \\ 7706 & 14.7 & 6.3 & 1.0 & \text { yes } & 0.40 \\ 7707 & 14.7 & 6.2 & 2.2 & \text { yes } & 0.60\end{array}$

4The coating used here was "Ultradel 1414" manufactured by Amoco Chemical Company. 


\section{DEVICE CHARACTERIZATION}

The LALC $\mathrm{Si}(\mathrm{Li})$ detectors were characterized at $80 \mathrm{~K}$ by measuring the detector leakage current and capacitance as functions of applied voltage, and in response to $2-60 \mathrm{keV}$ photons. Typical values for detector leakage current, as a function of reverse bias voltage, are shown in Fig. 2. The leakage currents are slightly higher than those typically seen in conventional $\mathrm{Si}(\mathrm{Li})$ devices, but are well within an acceptable operating range. Several of the detectors were thermally cycled between $80 \mathrm{~K}$ vacuum and room temperature air numerous times, without suffering any degradation in leakage current or spectral performance.

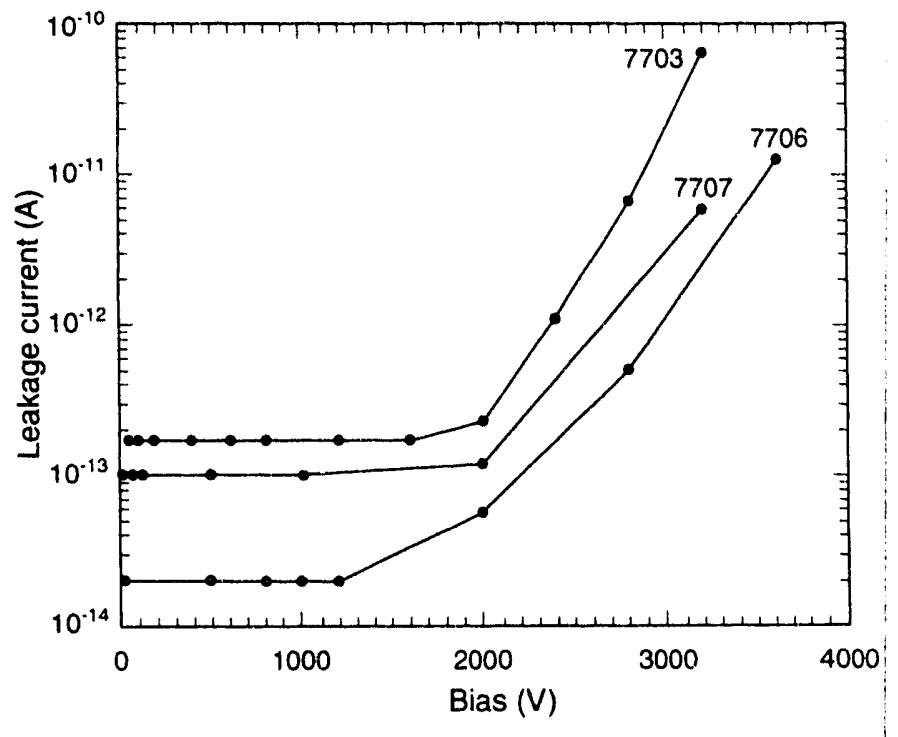

XBL 923.5239

Fig. 2 Leakage current, as a function of detector reverse bias, for typical LALC $\mathrm{Si}(\mathrm{Li})$ detectors.

The detectors depleted at less than $60 \mathrm{~V}$ reverse bias, as shown in the capacitance-voltage curves of Fig. 3. The fullydepleted detector capacitance depended predominantly on the anode diameter, as shown in Fig. 4, but also depended slightly on the detector diameter and thickness. For example, detector $\# 7706$, with an anode diameter of $1.0 \mathrm{~mm}$, thickness of 6.3 $\mathrm{mm}$ and outside diameter of $14.7 \mathrm{~mm}$, had a measured capacitance of $0.4 \mathrm{pf}$, compared to a conventional planar device with an equivalent thickness and diameter with a capacitance of 2.7 pf. The LALC detector capacitance can be estimated using the equation for the capacitance, $\mathrm{C}$, of a coaxial hemisphere [5]:

$$
C=\frac{2 \pi \varepsilon \varepsilon_{0}(R r)}{(R-r)}
$$

where $\varepsilon$ is the relative dielectric constant for $S i, \varepsilon_{0}$ is the free space permittivity constant, $R$ is the radius of the outer hemisphere (replaced by the thickness of the detector in this case), and $\mathrm{r}$ is the radius of the inner coaxial hemisphere (replaced by $3 / 4$ of the anode radius for the LALC detector in this case, because the flat anode has a smaller area than a hemisphere of equivalent radius). The measured data for the detectors deviates from this equation for anode diameters less than approximately $1.5 \mathrm{~mm}$, due to the stray capacitances in the test spectrometer which begin to dominate at $<0.5 \mathrm{pf}$.

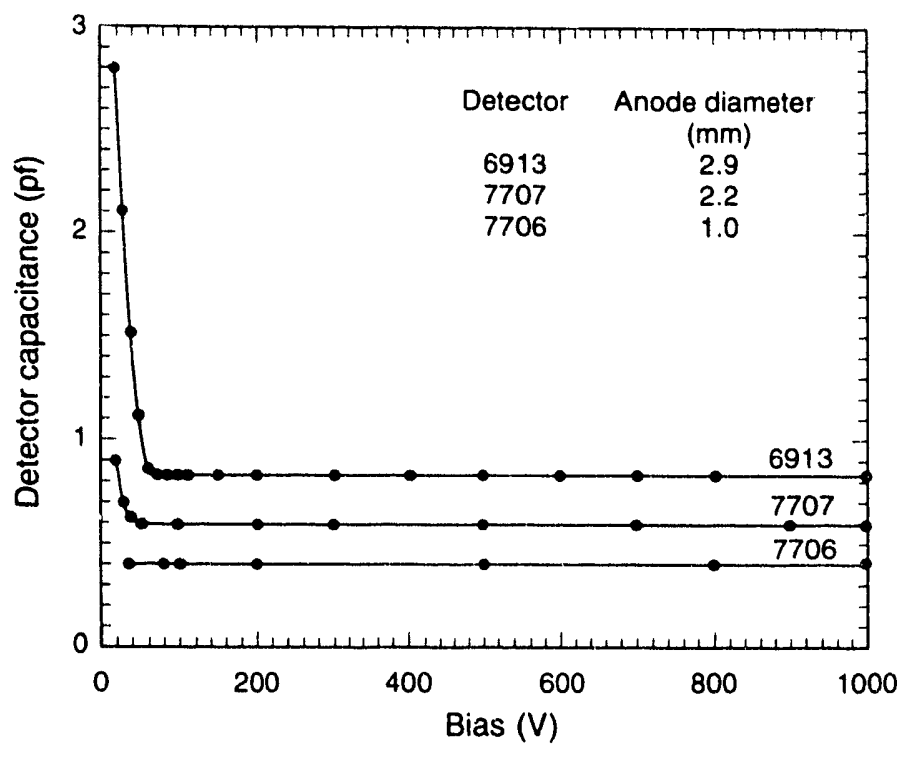

XBL 923.5238

Fig. 3 Detector capacitance, as a function of reverse bias, for three LALC $\mathrm{Si}(\mathrm{Li})$ detectors. The fully-depleted detector capacitance depends predominantly on the detector anode diameter.

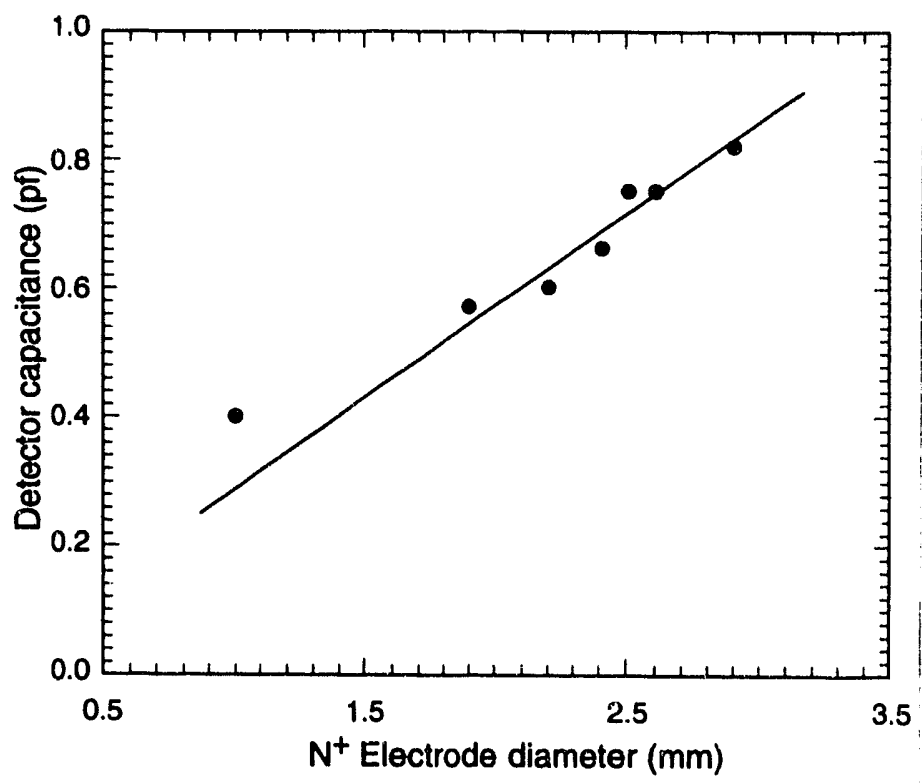

XBL $023-5237$

Fig. 4 Measured detector capacitance, as a function of anode diameter, for the LALC $\mathrm{Si}(\mathrm{Li})$ detectors. The line is equation (2) from text. 
The measured and calculated electronic noise, as a function of amplifier peaking time, arc shown in Fig. 5 for a $0.5 \mathrm{pf}$ LALC detector in combination with a 3.5 pf FET. The measured noise deviates slightly from the calculated noise at longer peaking times, due to the predominance of the $1 / f$ noise component at longer peaking times. Also shown in Fig. 5, for comparison purposes, are the calculated noise curves for an equivalent area conventional $\mathrm{Si}(\mathrm{Li})$ detector of $3 \mathrm{pf}$, and the calculated low noise that could be achieved if a lower capacitance FET was matched with one of the LALC Si(Li) detectors. It is our intent to use the LALC Si(Li) detectors in conjunction with 2 pf FETs for optimum $x$-ray spectrometer performance.

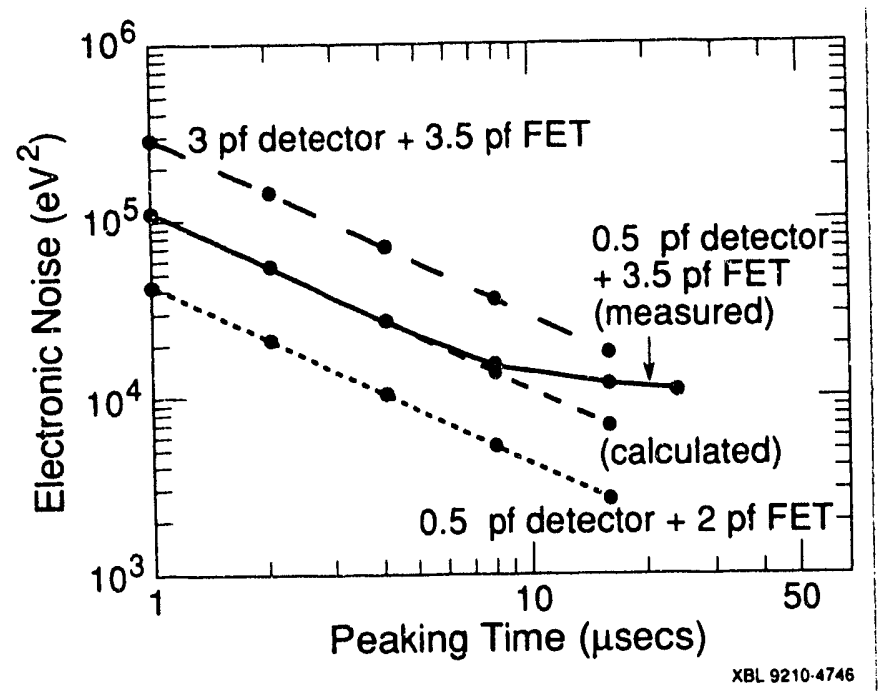

Fig. 5 Measured and calculated electronic noise, as a function of amplifier peaking time, for two LALC detectors and a conventional detector with an equivalent area as the LALC detectors.

\section{DETEC'TOR X-RAY RESPONSE}

Figure 6 shows the spectral response of two LALC detectors, one with a $45^{\circ}$ bevel and one without the bevel, to an $\mathrm{Fe}^{55}$ source. The source was collimated to $1 \mathrm{~mm}$ on the outer periphery of each of the detectors. The detector without the bevel shows very poor charge collection at the detector periphery due to a weak electric field in the corner regions, while the charge collection at the periphery of the detector with the bevel is comparable to that of a conventional device. Figure 7 shows that the charge collection and spectral quality at the periphery of a beveled detector is almost identical to that at the center of the same device. Our computer simulations modeling the electric field distribution in reverse-biased LALC detectors showed that the corner regions of the p-contact surface for detectors without the $45^{\circ}$ bevel were indeed areas of very weak field. These computer simulations predicted that very large biases, well beyond the typical breakdown values for these types of devices, would be required to fully deplete the

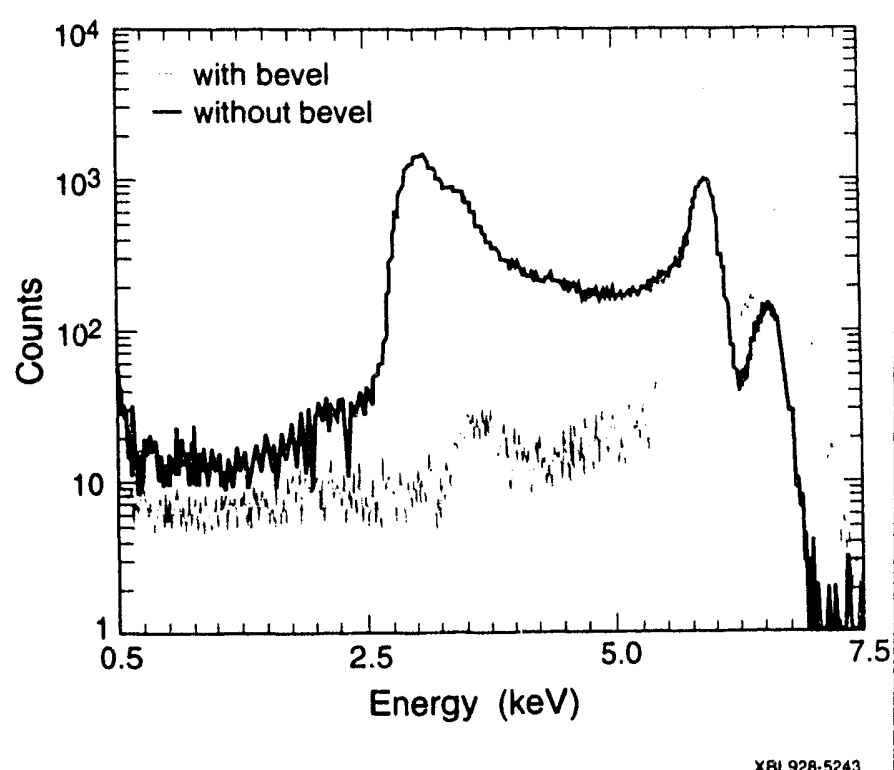

Fig. $6 \mathrm{Fe}^{55}$ spectra for two LALC detectors. The solid curve is the spectra for a detector without a $45^{\circ}$ bevel, with the $\mathrm{Fe}^{55}$ source collimated to $1 \mathrm{~mm}$ on the periphery of the detector. The dashed curve is a similar spectra, taken under identical conditions, except the detector has a $45^{\circ}$ bevel. Both spectra were taken for equal amounts of time.

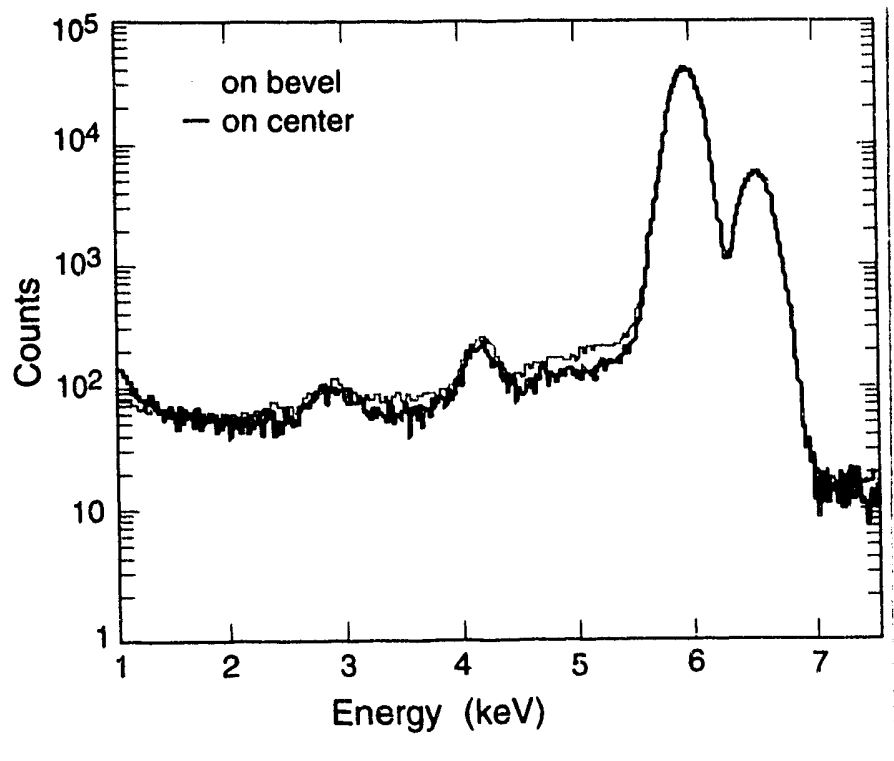

XBL928.5276

Fig. $7 \mathrm{Fe}^{55}$ spectra taken with a $1 \mathrm{~mm}$ collimated source at the center (solid line) and at the edge (dashed line) of a beveled LALC detector, normalized to the $5.9 \mathrm{keV}$ peak.

corner regions of a device without the bevel. For example, an LALC $\mathrm{Si}(\mathrm{Li})$ detector with a $14 \mathrm{~mm}$ outside diameter and a thickness of $6 \mathrm{~mm}$ would require approximately $4000 \mathrm{~V}$ to deplete within $0.5 \mathrm{~mm}$ of the corner. The introduction of the 2 $\mathrm{mm}$ deep $45^{\circ}$ bevel essentially eliminates the low ficld regions 
and good charge collection throughout the device can occur at less than half that voltage. Even with the bevel, however, detectors with diameters greater than approximately $16 \mathrm{~mm}$ require bias voltages in excess of $2000 \mathrm{~V}$ for adequate charge collection in all regions of the device. The unacceptable increase in detector leakage current at voltages greater than $2000 \mathrm{~V}$ places a practical limit on the diameter of these devices at approximately $16 \mathrm{~mm}$.

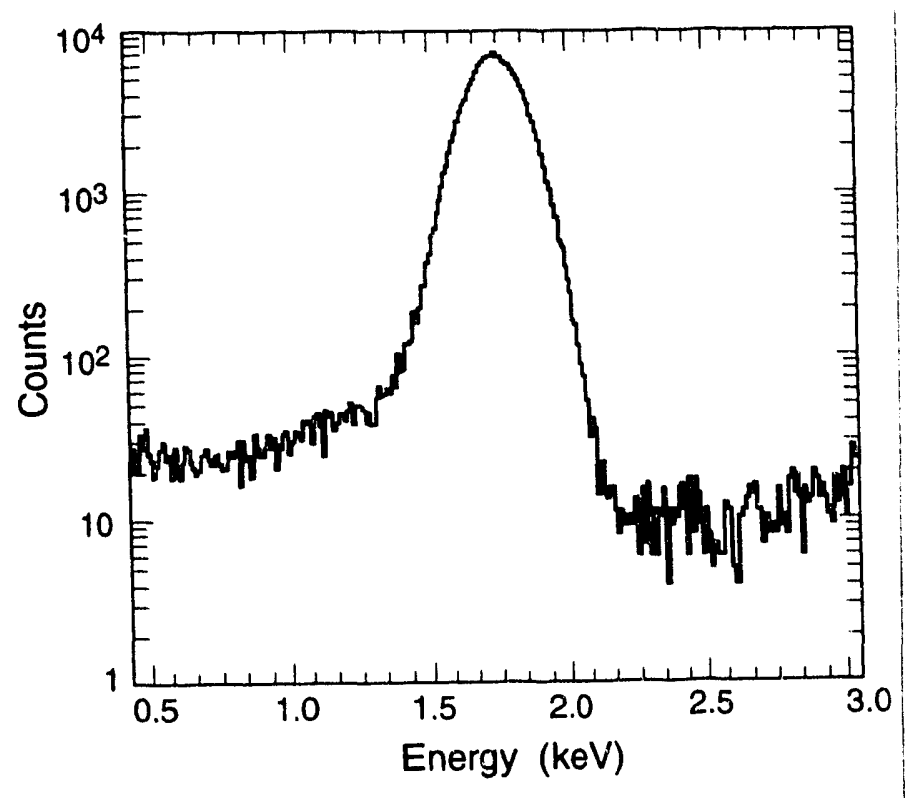

XBL.928.5278

Fig. 8 Spectral response of an LALC detector, with a Pd surface barrier entrance window contact, to $1.77 \mathrm{kvV} \mathrm{x}$-rays.

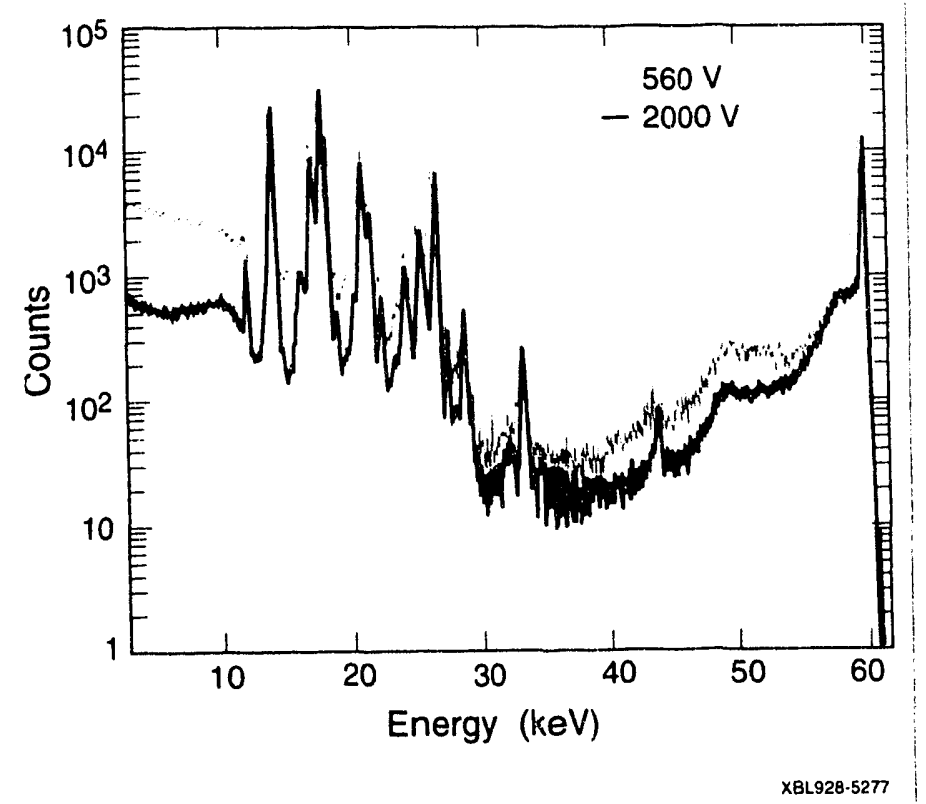

Fig. $9 \mathrm{Am}^{241}$ spectra for an LALC detector at $560 \mathrm{~V}$ bias (dashed line) and at $2000 \mathrm{~V}$ bias (solid line).
The low energy charge collection properties and detector dead layer effects are illustrated in the $1.77 \mathrm{keV}$ spectrum of Fig. 8. The detector dead layer is very thin, as is indirectly shown by the symmetry of the $1.77 \mathrm{keV}$ peak, and is identical to that of a conventional $\mathrm{Si}(\mathrm{Li})$ detector with a similar $\mathrm{Pd}$ surface barrier entrance window contact [6].

The effect of detector bias on spectral quality is demonstrated in the $\mathrm{Am}^{241}$ spectra of Fig. 9. While approximately $1000 \mathrm{~V}$ bias on a $6 \mathrm{~mm}$ thick detector is sufficient for adequate charge collection of the lower energy photons from an $\mathrm{Fe}^{55}$ source, higher energy photons from an $\mathrm{Am}^{241}$ source, which go deeper into the bulk of the detector, require higher electric fields ( $2000 \mathrm{~V}$ on a $6 \mathrm{~mm}$ thick device) for good charge collection. This is most likely due to weak field regions at the back surface of the detector, although our computer simulations of the electric field distribution in the detectors did not specifically show this. Additional computer modelling, more sophisticated than what we have used to date, is required to resolve the questions concerning the charge collection anomalies seen in these devices. However, given adequate bias, the $\mathrm{Am}^{241}$ spectral quality of an LALC Si(Li) detector is as good as that of a conventional $\mathrm{Si}(\mathrm{Li})$ device, as is shown in Fig. 10.

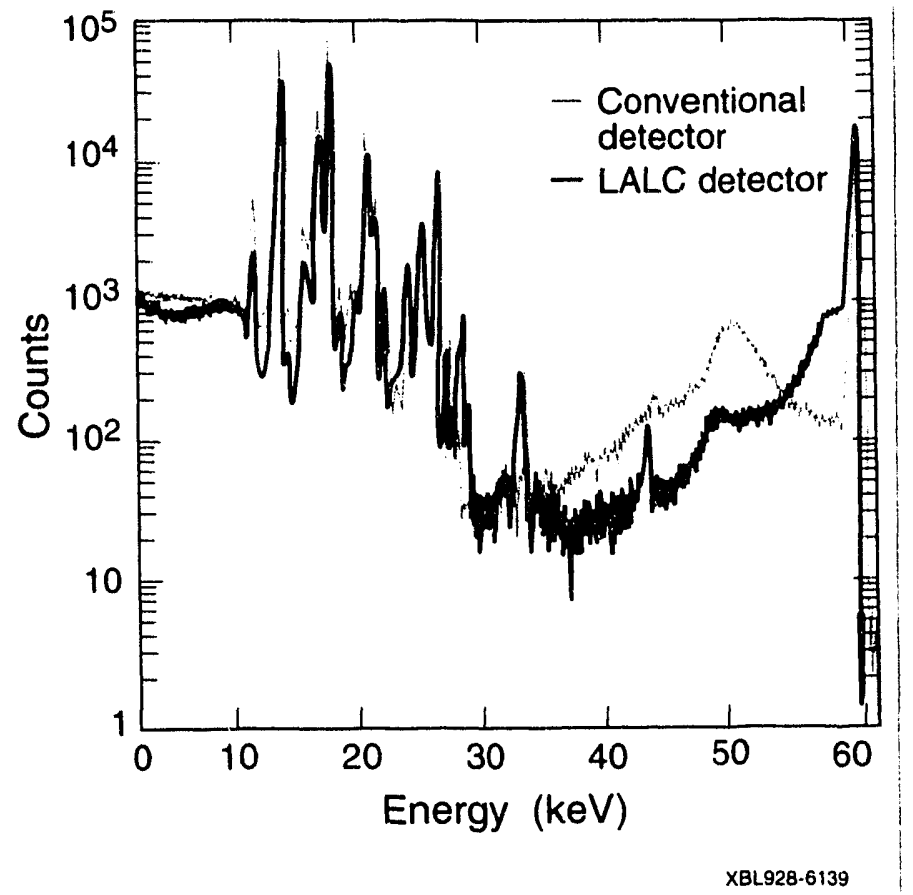

Fig. $10 \mathrm{Am}^{241}$ spectra for a conventional detector (dashed line) and an LALC detector (solid line), normalized to the 59.5 $\mathrm{keV}$ peak. The difference in the Compton scattering background from the $59.5 \mathrm{keV}$ peak is due to the difference in thickness of the two detectors $(4 \mathrm{~mm}$ thick conventional detector, $6 \mathrm{~mm}$ thick LALC detector). 


\section{DISCUSSION AND CONCLUSIONS}

The LALC Si $(\mathrm{Li})$ detectors developed here exhibit the same spectral quality as state-of-the-art conventional $\mathrm{Si}(\mathrm{Li})$ detectors for high energy resolution applications, but have the additional advantages of having large areas (up to approximately 200 $\left.\mathrm{mm}^{2}\right)$, but very small capacitances $(\neg 0.5 \mathrm{pf})$ to maintain low noise and excellent energy resolution. Unlike with conventional detectors, the LALC detectors do not require collimation to remove the anomalous charge collection characteristics sometimes seen at the periphery of conventional detectors [7], nor do they have the dead volume of the outer periphery of the conventional "grooved" structure, which allows for close packing of the LALC detectors into array structures. The LALC detectors can be best optimized by combining them with low capacitance FETs for use in spectrometers capable of operating at higher count rates than conventional spectrometers without compromising energy resolution. Conversely, the spectrometers can be run at standard count rates, but with improved energy resolution. For example, a 0.5 pf LALC detector combined with a typical 4 pf FET can run 2.5 times faster than an equivalent conventional system without sacrificing energy resolution. However, for optimum performance, the LALC detector should be matched with a low capacitance FET which will result in a system capable of running 8-10 times faster than a conventional system. We are currently in the process of building two spectrometers, with 2 pf FETs, for use with the LALC detectors: One is designed for a synchrotron microprobe application and one for a synchrotron EXAFS application. Spectrometer and detector performance, as related to these two applications, will be reported in future publications.

\section{DISCLAIMER}

Reference to a company or product name does not imply approval or recommendation of the product by the University of California or the U.S. Department of Energy to the exclusion of others that may be suitable.

\section{REFERENCES}

[1] F.S. Goulding and D.A. Landis, "Semiconductor detector spectrometer electronics," in Nuclear Spectroscopy and Reactions, Part A, J. Cerny, eds. New York: Academic Press, 1974 pp. $413-481$

[2] D.A. Landis, W.Y. Wong, J.T. Walton and F.S. Goulding, "Computer controlled drifting of $\mathrm{Si}(\mathrm{Li})$ detectors," IEEE Trans. Nucl. Sci., vol. NS-36, pp. 185-189, Feb. 1989.

[3] F.L. Allsworth, J.H. Howes and G. White, "Intrinsic germanium detectors used in a high counting rate system," Nucl. Instr. Meth., vol. 193, pp. 57-61, Feb. 1982.

[4] J.H. Howes and F.L. Allsworth, "High resolution X - gammaray spectrometry at high counting rates using high purity germanium detectors," IEEE Trans. Nucl. Sci., vol. NS-33, pp. 283-287, Feb. 1986.

[5] W.D. Cockrell, ed., Industrial Electronics Handbook, First Edition, New York: McGraw-Hill Book Co., Inc., 1958.

[6] C.S. Rossington, J.T. Walton and J.M. Jaklevic, "Si(Li) detectors with thin dead layers for low energy $x$-ray detection," IEEE Trans. Nucl. Sci., vol. NS-38(2) pp. 239243, April 1991.

[7] See, for example: J.L. Campbell, R.G. Leigh and W.J. Teesdale, "Peripheral imperfections and their effects on efficiency in $\mathrm{Si}(\mathrm{Li})$ x-ray detectors," Nucl. Instr. Meth., vol. B5, pp. 39-43, Sept.-Oct. 1984. 

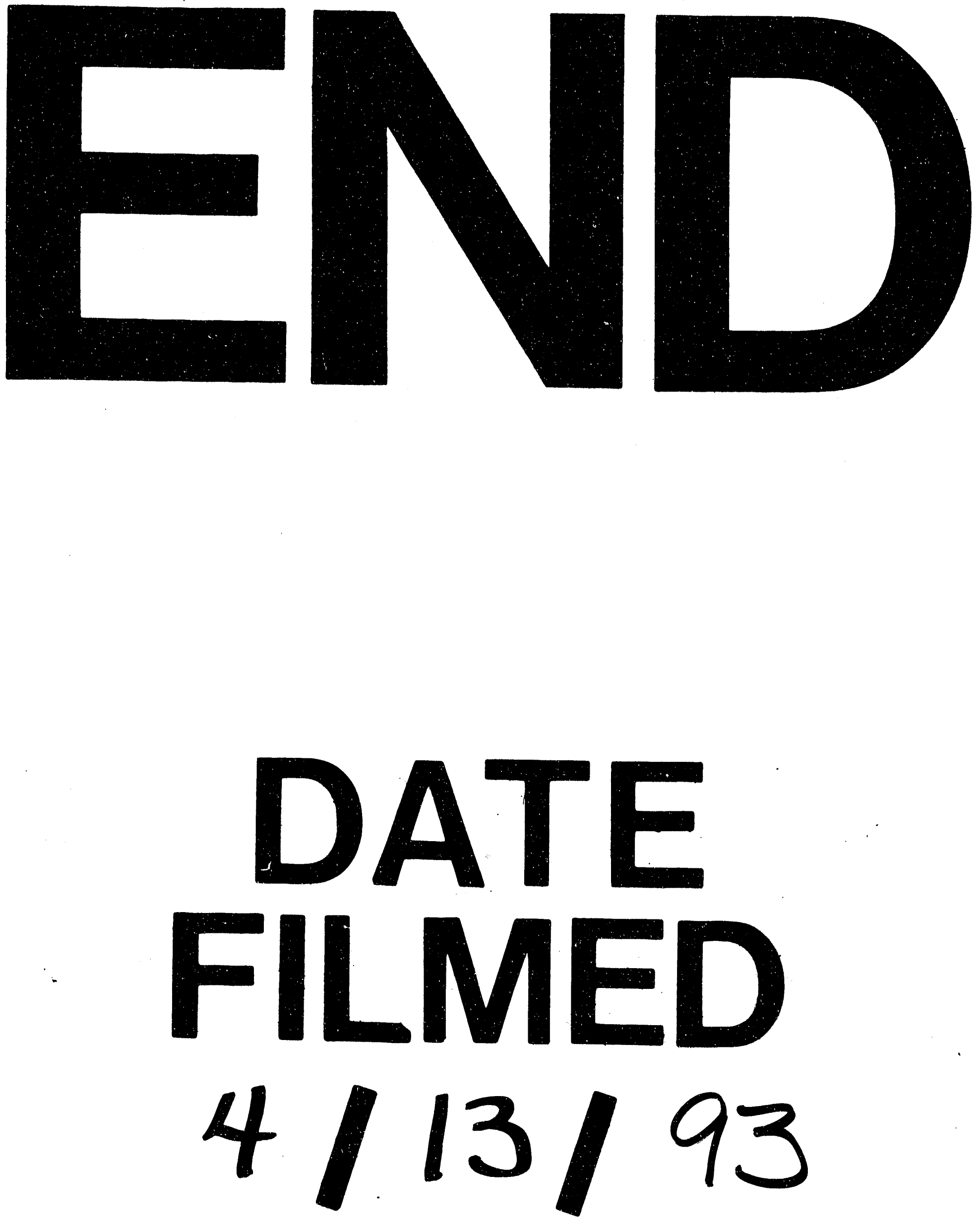
